Published in final edited form as:

Curr Opin Cell Biol. 2008 June ; 20(3): 310-315. doi:10.1016/j.ceb.2008.04.005.

\title{
CRACKING THE COREGULATOR CODES
}

\author{
Bert W. O'Malley", Jun Qin ",\#, and Rainer B. Lanz* \\ *Dept. of Molecular and Cellular Biology, Baylor College of Medicine, Houston, TX 77030 \\ \#Dept. of Molecular Biology and Biochemistry, Baylor College of Medicine, Houston, TX 77030
}

\section{Abstract}

The study of the genetic code has collectively revealed that the biochemical basis of heredity is uniform for nearly all known forms of life. Genetic approaches have generated a much better appreciation and understanding of many aspects of biological processes - and in some cases provided strategies for the treatment of human diseases. Still, the enormous and undoubtedly impressive amount of information gathered on gene sequences, their myriad expression patterns and translation into proteins is insufficient to answer seemingly simpler questions such as to what sets us humans apart from much more undemanding species while sharing almost the same sets of genes. Regulation of the proteome by posttranslational modifications is beginning to be understood as a major contributing factor to the structural and functional diversity in biology and for defining cellular mechanisms in particular. Covalent, posttranslational modifications provide an astonishingly rich and specific basis for an ultra-fast regulation of cellular processes, many of which converge to transcription units to control gene expression. With this essay we intend to share with the reader the rapid growth of our knowledge of the many conjunctions that exist between posttranslational modifications and key cellular processes that have emerged by studying the nuclear receptors and their transcriptional coregulators.

\section{INTRODUCTION}

Transcriptional coregulators comprise a heterogeneous and functionally distinct group of molecules that associate with transcription factors by direct binding or in the context of multi-protein complexes and integrate cellular signals by tethering varied enzymatic activities to the transcription unit for the purpose of regulating target gene expression. The entirety of this transcriptional regulation is coactivation or corepression. Coregulator functions include the transient modification of chromatin for transcription factor binding to DNA, the mediation and regulation of proximal and distal components of the transcription 'apparatus' including transcription initiation, elongation and splicing, but also the transduction and refinement of cellular signals to the locus of transcription as well as deceptively 'transcription-unrelated' cellular processes such as protein degradation or energy homeostasis in the mitochondria. See the following references for reviews on actions of NR coregulators in integrating signal-dependent programs of transcriptional responses at the molecular level $[1,10,17,26]$. Because of their broad functional activities, coregulators are the preferred class of target molecules to coordinately elucidate processes of cell regulation.

The work on nuclear receptor (NR) as recipients of coregulator signaling and mediators of transcription control has unveiled the regulatory potential of posttranslational modification (PTM) of the biological processes involved, suggesting that examining their diversity is critical for understanding mechanisms of cell regulation. To characterize the coregulator complexome - a cross-talking network of transcription factors, coregulators and their regulatory enzymatic molecules - a large-scale proteomics study that involves immunoprecipitation and mass spectrometry has been initiated (www.NURSA.org). The 
later provides a fundamental framework for detecting and mapping covalent modifications in proteins and quantifying their changes. Because PTMs are key to the physiologic functions of the proteins involved, it is imperative that we understand the 'coding' that these modifications impart to their complex components for their diverse activities. We therefore set out to 'crack' this coregulator code, which is to convert the PTM signals back into information that is understandable in the context of known cellular processes, and by doing so, to possibly unveil a system of principles or rules imposed by the modifications. The task is comprehensive and requires a methodical elucidation of the nature and the location of the modifications on key coregulator molecules, a determination of the composition of the complexes in which they reside, and confirmation of the diverse functions that PTMs impart on cell signaling and control of transcription. In addition to thorough proteomics approaches, this effort requires genome-wide functional analyses and sound bioinformatics strategies.

\section{DISCUSSION}

To date, the work on nuclear receptor coregulators has raised a series of important questions as to how a single primary protein can act in many diverse ways in the mammalian cell. For example, how does a coactivator become 'activated' to form distinct multimeric coactivator complexes for cellular functions? How does a coactivator differentially bind to NRs (or other transcription factors) for transcriptional activation at select promoters? How does a coactivator direct distinct subreactions of transcription (e.g., chromatin remodeling, transcriptional initiation, RNA chain elongation, RNA splicing, termination, etc.)? How can one coactivator participate in regulating molecular substeps of transcription, and then function in totally different cellular compartments to control mRNA translation [2], mitochondrial biology [3], or membrane initiated cell motility [4]? How does the same coregulator function as a 'coactivator' in certain instances, and as a 'corepressor' in other instances [5]? Perhaps even more perplexing, how can a specific coactivator function as an oncogene in certain cell contexts and a tumor suppressor in others [6,7]?

The molecular answer to the above series of questions is now becoming clear from recent work in the NR/coregulator and related fields. Logical interpretations of limited available data from a variety of sources suggest that the key to understanding the diversity of coregulator functions is an unexpectedly extensive posttranslational 'coding' of the coregulator proteins $[8,9]$. Such covalent modifications include, but are not limited to, phosphorylation, methylation, ubiquitylation, sumoylation and acetylation. In the case of coregulators, it has been observed that a combination of such modifications can lead to functionally distinct activities for the same primary sequence protein. The 'differentially coded' coactivator is now predisposed to form different multimeric coactivator complexes, and thereby, directed to interact with distinct genes and even to perform different compartmental functions in a cell $[2,10]$. Because such PTMs are the key to the physiologic functions of these proteins, it is imperative that we understand the 'coding' that these modifications impart to the coregulators for their diverse functions, and associate the kinases and enzymes that impart the PTM coding signals to the proteins in different cell types, and learn about the relationships of these coregulator modifications in the context of human pathologies.

Although known to exist for many decades, posttranslational modifications of proteins only recently have been subject to intensive study in terms of their regulatory potential. The power of phosphorylation was realized decades ago, beginning with studies of enzymes that demonstrated increased enzymatic activity when phosphorylated in response to small molecules [11]. The era of phosphokinase discovery further popularized the role of phosphorylation and that reaction dominated our thinking about regulatory protein 
modifications for a considerable period of time [12]. It is only recently that we have realized the critical role that multiple interdependent posttranslational modifications can play in the activation and deactivation of regulatory proteins [13]. Rough estimates suggest that $\sim 25,000$ human genes could produce $\sim 120,000$ primary sequence proteins after RNA splicing takes place. Of this total, at least $15 \%$ are likely to be transcriptional regulatory factors.

However, to focus on coregulators for this commentary, we need only consider a total of $\sim 300$ proteins (www.NURSA.org). The fact that many coregulators undergo extensive posttranslational modifications is no longer in question. So, if we accept that all coregulatory proteins contain PTMs, and that an average coregulator ( $\sim 80,000$ daltons in size) has $\sim 8$ different PTMs, an extremely conservative estimate, then the 'potential' for coregulator complex diversity in cells could be well in excess of one billion molecules per cellular gene [14]. It is highly unlikely that this potential is ever fully realized, and currently, there is no way of determining the exact amount of this potential ever used in a cell. However, since each single modification (PTM) can result in a protein with an altered function, it is an inescapable conclusion that multiple combinations of modifications can bestow a large array of distinct functions to any one regulatory protein. In reality, then, we potentially have an enormous number of different proteins within our body that can be used for the myriad of distinct regulatory functions required of mammals, and especially in humans.

PTMs can arise from transient interactions of coregulator complexes with enzymes in signaling pathways, or by intra-complex modifications by co-coregulators, most of which are themselves enzymes. The exact functional implications of PTMs of most coregulators are unclear at present. A few molecules, however, have been studied in greater detail. The SRC-3 (NCOA3; ACTR/AIB1/RAC3/pCIP/TRAM-1) coactivator is an example of one such molecule. Using proteomic approaches, over 40 separate modifications of the SRC-3 coregulator have been determined to date. These include phosphorylations, methylations, acetylations, ubiquitylations, sumoylations; in total, this plethora of PTMs creates enormous combinatorial potential for distinct functions in just this one coregulator.

To dissect the PTM coding within coactivators, it is necessary to methodically elucidate the nature and the location of the modifications on one molecule, to determine their function by mutational analyses, to determine the signaling enzymes/pathways responsible for their modifications, and to determine the interrelationships of modifications at one site on modifications occurring at more distant sites. This has not been done completely for any molecule yet but work is in progress. For example, phosphorylation of SRC-3 leads to formation of 'active' high molecular weight complexes with other coactivators, and the specific sites of phosphorylation can determine the co-coactivator partners that comprise the larger coactivator functional complexes [15]. Since coactivator complexes are dynamic, separate modifications then direct SRC-3 to dissociate from its partners in the coactivator complex. Examples of modifications inducing dissociation of SRC-3/ACTR coactivator complexes include acetylations and methylations $[9,16]$. In this way, rapid temporal enzymatic modifications promote a dynamic association and dissociation of active coactivator complexes. This equilibrium can be shifted by both ligands and signaling pathway modulations.

PTMs are not designed only for kinetic association/dissociation of coactivator proteins from their functioning complexes, but also are involved in activation/inactivation, assembly of specific partners in the functional complex, targeting specific transcription factors for regulation, and eventually for degradation of the coregulator proteins [10]. Coregulators are not primarily regulated at the level of DNA transcription, and that is logical since they appear to be the 'master genes' for regulating all other 'producer genes' [17]. If they were 
regulated solely by transcription, then they would require coregulators for their own genes, and then those coregulators would need other coregulators, and a never-ending circle would result. Consequently, coregulator levels usually are controlled primarily at the level of their degradation. PTMs are the effectors for this regulation and can either dictate more rapid degradation or prevent it, depending on the precise signaling enzymes involved [17]. PTMs are again at the heart of posttranslational regulation of coregulator degradation.

An activated coregulator complex must bind and regulate its appropriate target transcription factors. As one example of the combinatorial possibilities, inactive SRC-3 coactivator contains two key amino acids (aa.723; 786) that are sumoylated; they must be desumoylated for activation so that the molecule can bind with high affinity to the appropriate receptors. This event is followed by mono-ubiquitylations at these two sites which fully activates the molecule to bind to select NRs. These mono-ubiquitylations, however, are dependent upon two prior phosphorylations at separate downstream sites in the coactivator (aa. 505; 509), and by a specific kinase (GSK3). The mono-ubiquitylated sites are processively polyubiquitylated during subsequent rounds of transcription. When the ubiquitin chain reaches $4-5$ ubiquitins, it is then subject to binding by the $19 \mathrm{~S}$ cap of the proteasome and the protein undergoes degradation via the 26S proteasome [18] (Fig. 1). This example illustrates the combinatorial interplay of separate protein PTMs, each of which plays its own role, but their roles are interdependent. In terms of transcription, the results of the processive PTM ubiquitylations at amino acids 723/786 reveal the existence of a transcriptional 'time clock' which begins with activation of the coactivator molecule and ends with its proteosomal destruction [18]. We also found that peptidyl prolyl isomerase 1 (PIN1) catalyzes a conformational change of phosphorylated SRC-3 to promote its degradation [19]. On the other hand, phosphorylation of C-terminal residues of SRC-3 by atypical protein kinase $\mathrm{C}$ (aPKC), which is frequently overexpressed in cancers, stabilizes the coregulator in a selective ER-dependent manner by inhibiting its interaction with the $20 \mathrm{~S}$ proteasome core alpha C8 subunit, thereby promoting SRC-3 function and powerfully enhancing ER-dependent target gene transcription and estradiol-dependent cell growth [20]. These examples show a coordinate enzymatic organization of proteolytic pathways by different PTMs, using different cellular signals and enzymes (GSK3, PIN1, aPKC in these examples), strongly suggesting a fundamental importance for controlling cellular levels of key molecules such as SRC-3.

Clearly, SRC-3 is not the only coregulator of particular interest that has been shown to be subjected to PTMs for obtaining multivariate functions. PELP1, separately cloned and termed MNAR (Modulator of nongenomic activity of estrogen receptor), is a good example of a multitasking transcriptional coregulator that participates in both enhancement and repression of gene function and is regulated by PTMs [21]. Phosphorylation of PELP1/ MNAR for example, triggers estrogen activation of important signaling pathways such as the Src/MAPK and PI3K/Akt tracts to regulate cell proliferation and apoptosis [22,23]. NRinduced activation of specific intracellular kinases that control vital cellular functions has increasingly become a focus of NR/coregulator investigation. Such "nongenomic," or extranuclear, actions of NRs differ from the classical, transcription-dependent hormone action and involve the production of second-messenger molecules and the rapid activation of signal transduction pathways (e.g., ERK/MAPK, p38/MAPK, PI3K/AKT, PLC/PKC). These signaling cascades may occur in series or in parallel with NR-mediated transcription, and may cross-talk with other pathways, but converge at the level of modification of transcriptionally relevant molecules such as NRs and their coregulators. This synergistic coupling between extranuclear and genomic actions of hormones is accompanied by highly specific PTMs that serve as triggers but also as mediators and promoters of the signaling cascades. This scenario fundamentally revises the paradigms of classical cell signaling. In fact, covalent modifications are the only common trait the coregulators share in addition to 
transducing regulatory signals for the modulation of transcription. This also is true for the most unusual NR coregulator, the RNA, SRA [24], which is modified posttranscriptionally by the pseudouridine synthases Pus1p and Pus3p to trigger a coactivator-corepressor switch in the RNA [25], affording another means to regulate transcription.

A roundworm has $<1000$ cells but has a similar total gene count to humans. We have little genomic differences compared to mouse and other species. We are $99 \%$ similar to mouse but look at us!! How did we gain the proteome diversity that allows such differential evolutionary advancement compared to all other species? It is clear that the human species differs not just because of the number of different genes, but by how they are used. Humans have gained the capacity to multitask in terms of cell regulation. Multitasking leads to the grouping of gene expressions in a way so as to control major metabolic functions - and this requires proteome diversity. The coregulators are an example of such diversity; each PTM makes them different, and each mutation provides a distinction in function, and each distinction in function allows rapid regulation of multiple gene sets which have been evolutionarily grouped together to effect inter-compartmental regulation of physiologic processes. We believe that mammals and humans may have made a leap in evolution, in part, by accumulating combinations of modifiable amino acids and signaling pathway specializations within the coregulators. This approach permitted the evolution of gene groupings by leaps and bounds - and without much change in primary DNA and primary protein sequences. Moreover, if PTMs are playing such a role, then it follows that we humans also have the potential for unlimited future diversity without generation of new genes, but simply by adding more PTMs to our existing proteins to create new functions.

Since the coregulators can be considered to be 'master genes' that have evolved to coordinate multiple diverse cellular reactions to implement major physiologic processes [17], they also contain the potential to efficiently promote cellular pathologies by coordinately misdirecting multiple independent functions [26]. Evidence indicates that PTMs of coactivators can influence pathologic inflammation, aberrant adipogenesis, cell replication abnormalities and oncogenesis, etc. In fact, of the $\sim 300$ coregulators identified to date, $>165$ of them already have been associated with some disease state in humans [26] and (www.NURSA.org). Since most of these associations have been published only in the past 5 years, we suspect it represents only the tip of the iceberg of what remains to be learned for these master genes in terms of human diseases. Finally, since coregulator (and NR) PTMs are a result of prior enzymatic reactions, and thus, are subject to inhibition/activation with drugs, it is only a matter of time before the pharmaceutical industry realizes the potential of this new information for therapeutic intervention.

\section{CONCLUSION}

In conclusion, the recent advances in coregulator-controlled transcription and their extensive PTM patterns have opened opportunities to explore the intricate aspects of cell system biology in great detail. New knowledge such as that gained from the study of the SRC family of coregulators in our laboratory underline the key role that PTMs play as a cellular mechanism in providing and maintaining regulatory circuits so far not described in simpler organisms. While modern biology alone can't possibly suffice in offering plausible answers to the question of what makes us human, it certainly will provide a new framework in which other questions will find answers and new ideas for therapies for diseases, and provide a new platform for us scientists to concur and diverge on new hypotheses. Is the latter not an inherited human trait above all? 


\section{References}

1*. Rosenfeld MG, Lunyak VV, Glass CK. Sensors and signals: a coactivator/corepressor/epigenetic code for integrating signal-dependent programs of transcriptional response. Genes Dev. 2006; 20:1405-1428. A comprehensive review on the functional diversity of transcriptional coregulators and their ability to integrate cellular signals to generate precise, complex programs of gene expression. The signal-dependent, cyclic turnover of transcription factors and their associated coregulators is discussed in the context of epigenetic mechanisms. [PubMed: 16751179]

2*. Yu C, York B, Wang S, Feng Q, Xu J, O’Malley BW. An essential function of the SRC-3 coactivator in suppression of cytokine mRNA translation and inflammatory response. Mol Cell. 2007; 25:765-778. This paper describes a cytoplasmic function of SRC-3; newly discovered associations with the coregulator suggest that SRC-3 forms a complex with TIA-1 and promotes ARE-TIA-1 binding, translational silencing, and inhibition of TNF-a protein production. [PubMed: 17349961]

3. Handschin C, Spiegelman BM. Peroxisome proliferator-activated receptor gamma coactivator 1 coactivators, energy homeostasis, and metabolism. Endocr Rev. 2006; 27:728-735. A more recent review from the laboratory that originally cloned and characterized the first PGC-1 activator that, together with the homologues PGC-1 $\beta$ and PRC, play key roles in energy homeostasis, metabolism and cell fate determination. [PubMed: 17018837]

4. Joyce PL, Cox AD. Rac1 and Rac3 are targets for geranylgeranyltransferase I inhibitor-mediated inhibition of signaling, transformation, and membrane ruffling. Cancer Res. 2003; 63:7959-7967. [PubMed: 14633727]

5. Rogatsky I, Luecke HF, Leitman DC, Yamamoto KR. Alternate surfaces of transcriptional coregulator GRIP1 function in different glucocorticoid receptor activation and repression contexts. Proc Natl Acad Sci U S A. 2002; 99:16701-16706. By characterizing some features of GRIP1/ SRC-2 function and its interaction with GR on three different response elements, the authors provide the molecular evidences that collectively emphasize a remarkable context dependence of transcriptional regulation mediated by SRC-2 and GR. [PubMed: 12481024]

6*. Torres-Arzayus MI, Font de Mora J, Yuan J, Vazquez F, Bronson R, Rue M, Sellers WR, Brown M. High tumor incidence and activation of the PI3K/AKT pathway in transgenic mice define AIB1 as an oncogene. Cancer Cell. 2004; 6:263-274. This paper presents in vivo data that demonstrates that SRC-3 is able to act as an oncogene, since overexpression was sufficient to cause the formation of mammary carcinomas and other tumors in mice. [PubMed: 15380517]

7*. Coste A, Antal MC, Chan S, Kastner P, Mark M, O’Malley BW, Auwerx J. Absence of the steroid receptor coactivator-3 induces B-cell lymphoma. Embo J. 2006; 25:2453-2464. Also this work presents in vivo data; it shows that the deletion of the SRC-3 gene lead to thrombocytopenia and cell autonomous induction of lymphocyte proliferation, suggesting a selective antiproliferative action of SRC-3 in the hematopoietic system. [PubMed: 16675958]

8. Wu RC, Qin J, Yi P, Wong J, Tsai SY, Tsai MJ, O'Malley BW. Selective phosphorylations of the SRC-3/AIB1 coactivator integrate genomic reponses to multiple cellular signaling pathways. Mol Cell. 2004; 15:937-949. The authors of this paper show that phosphorylation of SRC-3 by IKK modulates SRC-3 activity and that SRC-3 can play distinct roles in diverse signaling pathways. [PubMed: 15383283]

9. Feng Q, Yi P, Wong J, O'Malley BW. Signaling within a coactivator complex: methylation of SRC-3/AIB1 is a molecular switch for complex disassembly. Mol Cell Biol. 2006; 26:7846-7857. In this paper, evidence is presented for SRC-3/AIB1 being methylated by CARM1 during estrogen signaling. This modification promotes dissociation of the SRC-3/CARM1 coactivator complex, thereby attenuating the transcriptional response and completing a dynamic equilibrium of receptormediated coactivator assembly and disassembly at the promoter. [PubMed: 16923966]

10. Lonard DM, O’Malley BW. The expanding cosmos of nuclear receptor coactivators. Cell. 2006; 125:411-414. [PubMed: 16678083]

11. Butcher RW, Ho RJ, Meng HC, Sutherland EW. Adenosine $3^{\prime}, 5^{\prime}$-monophosphate in biological materials. II. The measurement of adenosine $3^{\prime}, 5^{\prime}$-monophosphate in tissues and the role of the cyclic nucleotide in the lipolytic response of fat to epinephrine. J Biol Chem. 1965; 240:4515- 
4523. An original paper describing the involvement of cyclic AMP in the lypolytic response of the epididymal fat pad. [PubMed: 4378937]

12. Walsh DA, Perkins JP, Brosom CO, Ho ES, Kreb EG. Catalysis of the phosphorylase kinase activation reaction. J Biol Chem. 1971; 246:1968-1976. This work describes for the first time a specific site of action for cyclic AMP in a physiological process. [PubMed: 4323628]

13. Hunter T. The age of crosstalk: phosphorylation, ubiquitination, and beyond. Mol Cell. 2007; 28:730-738. A review on the multiple connections that exist between phosphorylation and ubiquitylation in the regulation of cellular processes. [PubMed: 18082598]

14. Lonard DM, O'Malley BW. Nuclear receptor coregulators: judges, juries, and executioners of cellular regulation. Mol Cell. 2007; 27:691-700. A review on NR coregulators with special emphasis on posttranslational modification codes. [PubMed: 17803935]

15. Wu RC, Qin J, Hashimoto Y, Wong J, Xu J, Tsai SY, Tsai MJ, O’Malley BW. Regulation of SRC-3 (pCIP/ACTR/AIB-1/RAC-3/TRAM-1) Coactivator activity by I kappa B kinase. Mol Cell Biol. 2002; 22:3549-3561. This paper describes the biochemical purification of SRC-1 and SRC-3 protein complexes and the identification of their associated proteins by mass spectrometry. SRC-3, but not SRC-1, was associated with the I kappa B kinase (IKK), and phosphorylation of SRC-3 by IKK was shown to modulate SRC-3 activity. [PubMed: 11971985]

16*. Xu W, Chen H, Du K, Asahara H, Tini M, Emerson BM, Montminy M, Evans RM. A transcriptional switch mediated by cofactor methylation. Science. 2001; 294:2507-2511. This work showes that protein methylation by CARM1 can regulate gene expression by providing a new molecular switch that determines whether CBP/p300 is to be used for NR-dependent or CREB-dependent gene activation. [PubMed: 11701890]

17. O’Malley BW. Molecular biology. Little molecules with big goals. Science. 2006; 313:1749-1750. [PubMed: 16990541]

18**. Wu RC, Feng Q, Lonard DM, O’Malley BW. SRC-3 coactivator functional lifetime is regulated by a phospho-dependent ubiquitin time clock. Cell. 2007; 129:1125-1140. This work provides the molecular evidence for proposing a "Ubiquitin Clock Theory" - a code of posttranslational modifications that determines the transcriptional potency and also the lifetime of SRC-3. [PubMed: 17574025]

19*. Yi P, Wu RC, Sandquist J, Wong J, Tsai SY, Tsai MJ, Means AR, O’Malley BW. Peptidyl-prolyl isomerase 1 (Pin1) serves as a coactivator of steroid receptor by regulating the activity of phosphorylated steroid receptor coactivator 3 (SRC-3/AIB1). Mol Cell Biol. 2005; 25:96879699. The authors demonstrate that Pin 1 interacts with phosphorylated SRC-3 to modulate its protein-protein interactions with other coregulators, thereby enhancing PR and ER function as well as directing the cellular turnover of SRC-3. [PubMed: 16227615]

20*. Yi P, Feng Q, Amazit L, Lonard DM, Tsai SY, Tsai MJ, O’Malley BW. Atypical protein kinase C regulates dual pathways for degradation of the oncogenic coactivator SRC-3/AIB1. Mol Cell. 2008; 29:465-476. Specific estrogen-mediated posttranslational modifications of SRC-3 can shield it from degradation by the proteasome, leading to increased SRC-3 accumulation and increased activity of downstream target genes such as those controlling cell growth, especially in hormone-responsive tumors. [PubMed: 18313384]

21. Vadlamudi RK, Wang RA, Mazumdar A, Kim Y, Shin J, Sahin A, Kumar R. Molecular cloning and characterization of PELP1, a novel human coregulator of estrogen receptor alpha. J Biol Chem. 2001; 276:38272-38279. [PubMed: 11481323]

22. Greger JG, Fursov N, Cooch N, McLarney S, Freedman LP, Edwards DP, Cheskis BJ. Phosphorylation of MNAR promotes estrogen activation of phosphatidylinositol 3-kinase. Mol Cell Biol. 2007; 27:1904-1913. [PubMed: 17194752]

23. Cheskis BJ, Greger J, Cooch N, McNally C, McLarney S, Lam HS, Rutledge S, Mekonnen B, Hauze D, Nagpal S, et al. MNAR plays an important role in ERa activation of Src/MAPK and PI3K/Akt signaling pathways. Steroids. 2008

24*. Lanz RB, McKenna NJ, Onate SA, Albrecht U, Wong J, Tsai SY, Tsai MJ, O’Malley BW. A steroid receptor coactivator, SRA, functions as an RNA and is present in an SRC-1 complex. Cell. 1999; 97:17-27. The original paper which, at a time of 'adolescence' of coregulator biology, has stressed many boundaries on how transcription can be controlled. [PubMed: 10199399] 
25. Zhao X, Patton JR, Ghosh SK, Fischel-Ghodsian N, Shen L, Spanjaard RA. Pus3p-and Pus1pdependent pseudouridylation of steroid receptor RNA activator controls a functional switch that regulates nuclear receptor signaling. Mol Endocrinol. 2007; 21:686-699. This paper demonstrates that functional 'switches' are not exclusive features of proteins, but can also regulate the RNA SRA. It is now accepted that functional 'switches' are an intrinsic property of the otherwise heterogeneous group of transcriptional coregulators. [PubMed: 17170069]

26. Lonard DM, Lanz RB, O'Malley BW. Nuclear receptor coregulators and human disease. Endocr Rev. 2007; 28:575-587. A review and catalogue of the involvement of coregulators in human diseases. [PubMed: 17609497] 


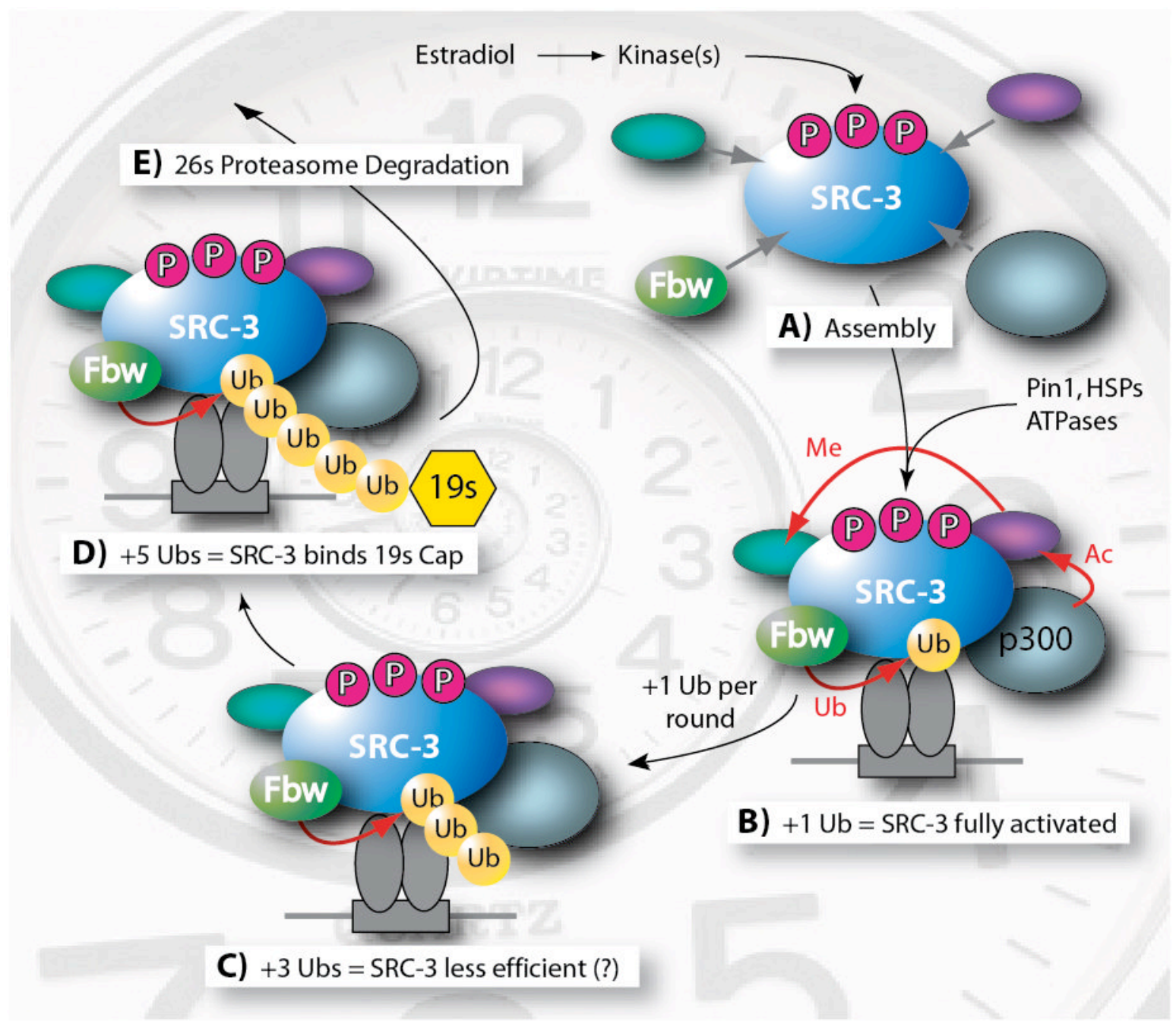

Figure 1.

(A) A cellular stimulus such as estrogen induces kinases to cause phophorylations of the SRC-3 coactivator that induce formation of a functional coactivator complex. Fbw (E3 ligase) enters complex and (B) mono-ubiquitylates SRC-3 to 'super-activate' it; other intracomplex modifications occur during the same time frame. (C) One additional ubiquitin molecule is added to SRC-3 during each round of transcription and the chain grows in number. (D) The polyubiquitin chain eventually extends to 5 ubiquitins. (E) At that point, the 19S Cap binds the polyubiquitylated coactivator and takes it to the $26 \mathrm{~S}$ proteasome for degradation. The time period from activation to degradation is $\sim 30$ seconds (if sequential) and represents a 'Ubiquitin-Dependent Transcriptional Time Clock' [14,18]. 